www.nature.com/pj

\title{
Conformational properties of a polymer tethered to an interacting flat surface
}

\author{
Hong Li ${ }^{1}$, Chang-Ji Qian ${ }^{1}$, Li-Zhen Sun ${ }^{2}$ and Meng-Bo Luo ${ }^{2}$ \\ The conformational properties of a lattice self-avoiding polymer chain tethered to an interacting and impenetrable flat surface \\ were simulated using a dynamic Monte Carlo method. The results show that the conformational size reaches a minimum at the \\ critical adsorption point (CAP) and that the scaling behavior of the polymer at the CAP is the same as that in the bulk solution. \\ The results provide a new method to determine the CAP of polymer chains.
}

Polymer Journal (2010) 42, 383-385; doi:10.1038/pj.2010.9; published online 17 March 2010

Keywords: critical adsorption point; Monte Carlo simulation; self-avoiding chain

\section{INTRODUCTION}

The adsorption of polymer chains on surfaces by means of physical or chemical interactions is an important subject in polymer and biological sciences. A polymer chain may adsorb or desorb, depending on interactions with the surface. This phenomenon is relevant to many technological applications such as in polymer compatibilizers, colloid stabilizers and polymeric surfactants. ${ }^{1-3}$ In many biological systems, ligands are attached to a surface through flexible tether chains. ${ }^{4,5}$ The conformation of the attached tether chains will affect the binding of the ligand to a receptor and will thus influence the whole biological process. ${ }^{6}$ The adsorption phenomenon is also a part of many physical systems; for example, polymer chains grafted to colloid particles and block copolymers at liquid-air interfaces. ${ }^{7}$ The adsorption of polymers has attracted a large number of theoretical and experimental studies. $^{7-12}$ The growing interest in polymers interacting with substrates warrants a thorough understanding of the static and dynamic properties of the tethered chain.

A mathematical model often used for studying the adsorption of tethered polymers on surfaces is the self-avoiding walk (SAW) chain in a three-dimensional (3D) simple cubic lattice that interacts with a flat surface and is restricted to lie on one side of the surface. ${ }^{13-17}$ Every walk step that contacts the surface is assigned an interaction energy $\varepsilon$ (in units of $k_{\mathrm{B}} T$, where $k_{\mathrm{B}}$ is the Boltzmann constant and $T$ is the temperature). It is well accepted that a single chain adsorbed on an attractive surface exhibits a phase transition from a desorbed state to an adsorbed state when the adsorption strength increases beyond a critical value. The CAP $\varepsilon_{\mathrm{c}}$ was estimated to be about -0.29 for polymers on the simple cubic lattice. ${ }^{9-12,16,18,19}$

In this work, we studied how the conformational properties of the chain change from a desorbed state for $\varepsilon>\varepsilon_{\mathrm{c}}$ to an adsorbed state for $\varepsilon<\varepsilon_{\mathrm{c}}$. We found that both the mean square end-to-end distance $\left\langle R^{2}\right\rangle$ and the mean square radius of gyration $\left\langle R_{\mathrm{G}}{ }^{2}\right\rangle$ have a minimum near $\varepsilon_{\mathrm{c}}$. The conformation size $\left\langle R^{2}\right\rangle$ and $\left\langle R_{\mathrm{G}}{ }^{2}\right\rangle$ increase rapidly when $\varepsilon<\varepsilon_{\mathrm{c}}$. The scaling law $\left\langle R^{2}\right\rangle \propto n^{2 v_{R}}$ holds for every interaction energy $\varepsilon$ we investigated. The exponent $v_{R}$ is dependent on $\varepsilon$. We found a minimum $v_{R} \approx 0.6$, equal to that in bulk solvent, at a CAP $\varepsilon_{\mathrm{c}} \approx-0.29$. This result provides a new method to determine $\varepsilon_{\mathrm{c}}$ in experiments and simulations.

\section{MODEL AND CALCULATION METHOD}

Simulations were performed on a simple cubic lattice. The flat surface was assumed to be infinitely large, impenetrable to the polymer chain, and located at $z=0$, with direction $z$ being perpendicular to the flat surface. The lattice layers above the surface were numbered $z=1,2, \ldots$ A SAW polymer chain comprises $n$ identical, consecutively linked monomers with a bond length of one lattice unit. The first monomer of the chain was fixed at position $(0,0,1)$ and was considered to be adsorbed to the impenetrable surface. We used a large simulation box to eliminate the size effect. The simulation sizes in the $x$ and $y$ directions were $L_{x}=L_{y}=n$, and periodic boundary conditions were used in the $x$ and $y$ directions. The length in the $z$ direction was $L_{z}=n$. Therefore, there was no size effect in the simulations.

At the beginning of the simulation, we grew a tethered SAW chain using the Rosenbluth-Rosenbluth method ${ }^{20}$ with the first monomer fixed at $(0,0,1)$. After creating a whole chain, we let it undergo a series of Brownian motions resulting from random collisions between chain beads and solvent molecules. The movement of the polymer chain was restricted to the upper half-space with $z>0$. The Brownian movement was achieved by a dynamic Monte Carlo technique that has been described in detail previously. ${ }^{21}$ In the dynamic model, a monomer is chosen randomly and one of the three elementary motions- the end-monomer, normal-monomer or $90^{\circ}$ crankshaft motion-is attempted. This trial move is accepted if the following three conditions are satisfied: (1) self-avoidance is obeyed, (2) the new site locates at $z>0$, and (3) the Boltzmann factor $\exp (-\Delta E)$ is greater than a random number uniformly distributed over the interval $(0,1)$, where $\Delta E$ (in unit of $k_{\mathrm{B}} T$ ) is the energy shift due to the

${ }^{1}$ Department of Physics, Wenzhou University, Wenzhou, China and ${ }^{2}$ Department of Physics, Zhejiang University, Hangzhou, China

Correspondence: Professor C-J Qian, Department of Physics, Wenzhou University, Wenzhou 325035, China.

E-mail: kjqian838@vip.sina.com

Received 23 November 2009; revised 18 January 2010; accepted 20 January 2010; published online 17 March 2010 
change in the monomer site. In one Monte Carlo step, $n$ monomers in the chain attempt to move once, on average.

The simulation was started from $\varepsilon=0$ with a desorbed state to $\varepsilon=-0.6$ with a highly adsorbed state by slowly decreasing $\varepsilon$. At each $\varepsilon$, the chain was relaxed for a time period $\tau=2.5 n^{2.13}$, which was found to be long enough to achieve an equilibrium state. ${ }^{22}$ In the present calculation, 10000 independent runs with different starting configurations and random number series were performed. All quantities were thus averaged over these 10000 runs.

\section{RESULTS AND DISCUSSION}

We calculated the mean-square end-to-end distance $\left\langle R^{2}\right\rangle$ at different interactions. Here, the end-to-end distance was $R=\left|\vec{r}_{n}-\vec{r}_{1}\right|$, where $\vec{r}_{1}$ and $\vec{r}_{n}$ are the position vectors of the first and last monomers. The dependence of $\left\langle R^{2}\right\rangle / n$ on the interaction $\varepsilon$ is plotted in Figure 1 for a variety of chain lengths. We found that each curve had a minimum. Interestingly, the minimum was located near the CAP $\varepsilon_{\mathrm{c}}=-0.29$ of the model system. ${ }^{12,16}$ Above $\varepsilon_{\mathrm{c}}\left\langle R^{2}\right\rangle$ increased slowly with $\varepsilon$, while below $\varepsilon_{\mathrm{c}}$ it grew rapidly with the decrease in interaction. The minimum $\left\langle R^{2}\right\rangle$ became more obvious with increasing chain length. This result indicates that one can locate $\varepsilon_{\mathrm{c}}$ from the mean-square end-to-end distance of a single long chain length, which is more convenient than the scaling method in which different chain lengths are needed. The end-to-end distance of a long chain length can be measured from the angular distribution of the intensity of the scattered light, which can be studied easily by experimentation. ${ }^{23}$

The mean-square radius of gyration $\left\langle R_{\mathrm{G}}{ }^{2}\right\rangle$ was also computed. The radius of gyration is defined as $R_{\mathrm{G}}^{2}=\frac{1}{n} \sum_{i=1}^{n}\left(\vec{r}_{i}-\vec{r}_{c}\right)^{2}$, where $\vec{r}_{i}$ and $\vec{r}_{c}$ are the position vectors of the $i$ th monomer and the mass center of the chain, respectively. The dependence of $\left\langle R_{\mathrm{G}}{ }^{2}\right\rangle$ on interactions is plotted for chain lengths $n=50,100,200$ and 400 in Figure 2. We found that $\left\langle R_{\mathrm{G}}^{2}\right\rangle$ behaved similarly to $\left\langle R^{2}\right\rangle$. The minimum of $\left\langle R_{\mathrm{G}}{ }^{2}\right\rangle$ was also near the CAP $\varepsilon_{\mathrm{c}}=-0.29$. However, the minimum of $\left\langle R_{\mathrm{G}}{ }^{2}\right\rangle$ was not as obvious as that of $\left\langle R^{2}\right\rangle$. Thus, we focus mainly on $\left\langle R^{2}\right\rangle$ in the following analysis.

It is well known that the conformation size scales with the chain length. For example, $\left\langle R^{2}\right\rangle$ is in a scaling law with the chain length $n$ according to the equation

$$
\left\langle R^{2}\right\rangle \propto n^{2 v_{R}}
$$

For a free SAW chain in a simple cubic lattice used as a model of the chain in bulk solution, the exponent $v_{R}$ is about 0.6. For a tethered polymer chain, the expected asymptotic behavior above the CAP $\left(\varepsilon>\varepsilon_{\mathrm{c}}\right)$ is the same as for polymers in bulk. At the CAP, the scaling behavior is also valid, even for finite chain lengths. ${ }^{16}$ For a small chain length $n$ and at $\varepsilon$ above the CAP, the perpendicular component $\left\langle R^{2}\right\rangle_{z}$ is influenced by the surface and the correction to scaling becomes important. ${ }^{16}$ At $\varepsilon=0,\left\langle R^{2}\right\rangle_{z}$ can be expressed as

$$
\left\langle R^{2}\right\rangle_{z}=A_{z} n^{2 v_{R}}\left(1+c / n^{\psi}\right),
$$

with $c<0$, found by assuming $v_{R}$ is the bulk value. ${ }^{16}$ The scaling behavior of the parallel component $\left\langle R^{2}\right\rangle_{x y}$ is not affected by the surface.

For a finite chain length, we have examined whether the scaling law in Equation (1) still exists for a tethered chain on an interacting surface. In this way, we assumed the exponent $v_{R}$ was not always the bulk value. The results are presented in the inset of Figure 3; such a scaling relation always exists at interactions above and below $\varepsilon_{\mathrm{c}}$. The dependence of $v_{R}$ on the interaction $\varepsilon$ is plotted in Figure 3. We found that $v_{R}$ also has a minimum near $\varepsilon_{\mathrm{c}}$. The minimum value is very close to 0.6 , a typical value for a SAW chain in bulk solution. This result is consistent with the idea that the chain at the CAP behaves in the same

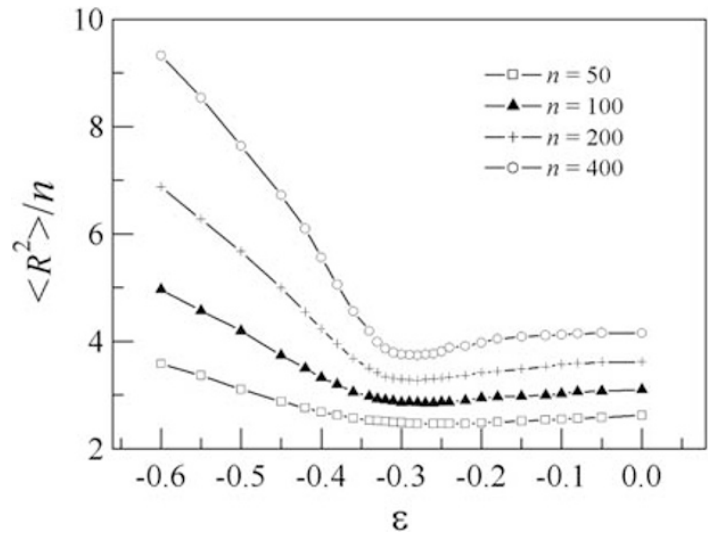

Figure 1 Mean-square end-to-end distance $\left\langle R^{2}\right\rangle$ vs interaction $\varepsilon$ for chain length $n=50,100,200$ and 400 .

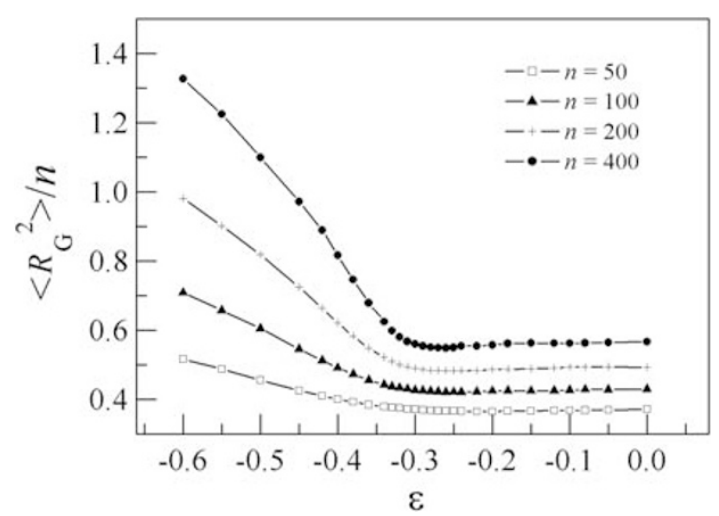

Figure 2 Mean-square radius of gyration $\left\langle R_{\mathrm{G}}{ }^{2}\right\rangle$ vs interaction $\varepsilon$ for chain length $n=50,100,200$ and 400 .

manner as a chain in bulk solution. ${ }^{14}$ This provides an alternative method to locate $\varepsilon_{\mathrm{c}}$

The dependence of the mean-square radius of gyration $\left\langle R_{\mathrm{G}}{ }^{2}\right\rangle$ on the chain length $n$ was also found to obey the same scaling law: $\left\langle R_{\mathrm{G}}{ }^{2}\right\rangle \propto n^{2 v_{S}}$. We found the behavior of the exponent $v_{S}$ to be similar to that of $v_{R}$. We also found that $v_{S}$ has a minimum at about $\varepsilon_{\mathrm{c}}=-0.29$.

In this model system, a chain exhibits a transition from a desorbed state at $\varepsilon>\varepsilon_{\mathrm{c}}$ to an adsorbed state at $\varepsilon<\varepsilon_{\mathrm{c}}$. The transition can clearly be seen from the behavior of the parallel and perpendicular components of $\left\langle R^{2}\right\rangle,\left\langle R^{2}\right\rangle_{x y}$ and $\left\langle R^{2}\right\rangle_{z}$, respectively. Figure 4 shows the dependence of $\left\langle R^{2}\right\rangle_{x y}$ and $\left\langle R^{2}\right\rangle_{z}$ on the interaction $\varepsilon$. At $\varepsilon=0$, $\left\langle R^{2}\right\rangle_{z}>\frac{1}{2}\left\langle R^{2}\right\rangle_{x y}$, indicating that the $z$ component is enlarged by the excluded volume effect of the surface. The decrease of $\left\langle R^{2}\right\rangle_{z}$ and the increase of $\left\langle R^{2}\right\rangle_{x y}$ at $\varepsilon<0$ show that the chain tends to approach the surface because of the attraction of the surface. $\left\langle R^{2}\right\rangle_{z}$ tends to zero for $\varepsilon<\varepsilon_{\mathcal{c}}$, which means that the chain is very close to the surface and is almost two-dimensional. The value of $v_{R}$ at $\varepsilon=-0.6$ is quite close to 0.75 , the value of a two-dimensional SAW chain. ${ }^{24}$ Our results reveal a conformational transition of the polymer from a threedimensional state to a two-dimensional state at $\varepsilon_{\mathrm{c}}$.

We found that a rapid decrease in $\left\langle R^{2}\right\rangle_{z}$ occurs at $\varepsilon_{\mathrm{c}}=-0.29$, as shown in Figure 4. It is clear that the decrease in $\left\langle R^{2}\right\rangle_{z}$ at $\varepsilon_{\mathrm{c}}=-0.29$ was larger than the increase in $\left\langle R^{2}\right\rangle_{x y} .\left\langle R^{2}\right\rangle_{x y}$, however, increased very fast below $\varepsilon_{\mathrm{c}}$. Therefore, one can find a minimum conformation size near $\varepsilon_{\mathcal{c}}$, as shown in Figures 1 and 2 . 


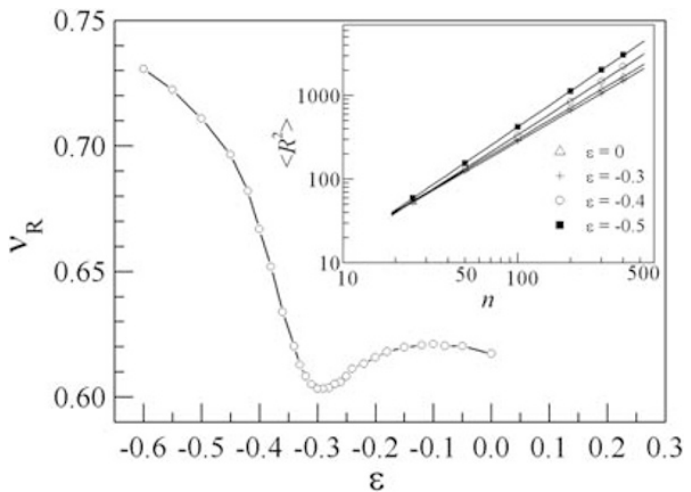

Figure 3 Plot of the exponent $v_{R}$ vs the interaction $\varepsilon$. The inset presents the log-log dependence of the mean-square end-to-end distance $\left\langle R^{2}\right\rangle$ on the chain length $n$ at different interactions: $\varepsilon=0,-0.3,-0.4$ and -0.5 . The solid lines in the inset are linear fits.

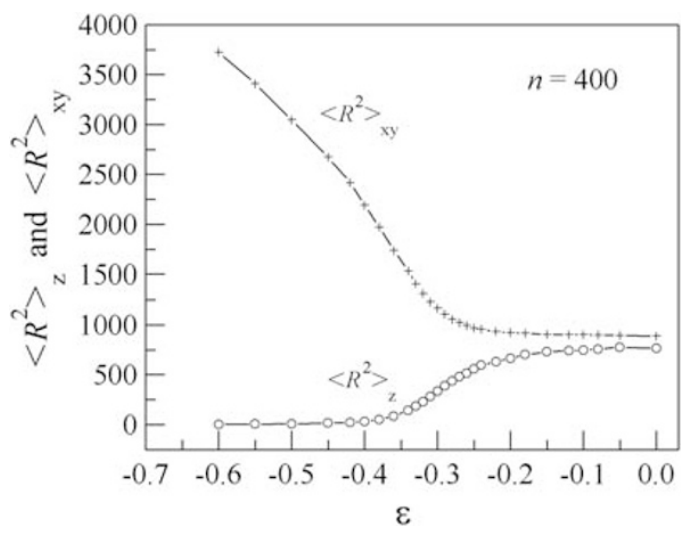

Figure 4 Plot of $\left\langle R^{2}\right\rangle_{x y}$ and $\left\langle R^{2}\right\rangle_{z}$ against the interaction $\varepsilon$ for chain length $n=400$.

\section{CONCLUSION}

Dynamic Monte Carlo simulations were carried out for a lattice selfavoiding polymer chain with one end tethered to an interacting, flat surface. The conformational properties of the tethered chain were dependent on the polymer-surface interaction $\varepsilon$. In the vicinity of the CAP $\varepsilon_{\mathrm{c}}=-0.29$, the conformational size $\left\langle R^{2}\right\rangle$ and $\left\langle R_{\mathrm{G}}{ }^{2}\right\rangle$ were minimized. The scaling exponent $v_{R}$ also reached a minimum near $\varepsilon_{\mathrm{c}}$. The minimum $v_{R}$ was about 0.6 , the same as that of a chain in bulk solution. The chain transitioned from a three-dimensional extended state to a two-dimensional adsorbed state at $\varepsilon_{\mathrm{c}}$. Our results provide an alternative way to determine the CAP.

\section{ACKNOWLEDGEMENTS}

This work was supported by the National Natural Science Foundation of China (Grant No. 20771092) and the Natural Science Foundation of Zhejiang Province, China (Grant No. Y607142).

1 Milner, S. T. Polymer brushes. Science 251, 905 (1991)

2 Alexander, S. Adsorption of chain molecules with a polar head a scaling description. J. Phys. (Paris) 38, 983 (1977).

3 de Gennes, P. G. Conformations of polymers attached to an interface. Macromolecules 13, 1069 (1980).

4 Wong, J. Y., Kuhl, T. L., Israelachvili, J. N., Mullah, N. \& Zalipsky, S. Direct measurement of a tethered ligand-receptor interaction potential. Science 275, 820 (1997).

5 Jeppesen, C., Wong, J. Y, Kuhl, T. L., Israelachvili, J. N., Mullah, N., Zalipsky, S. \& Marques, C. M. Impact of polymer tether length on multiple ligand-receptor bond formation. Science 293, 465 (2001).

6 Xue, B. \& Wang, W. Influence of external vibration on tether chain in ligand-receptor binding. J. Chem. Phys. 122, 194912 (2005).

7 Luettmer-Strathmann, J., Rampf, F., Paul, W. \& Binder, K. Transitions of tethered polymer chains. J. Chem. Phys. 128, 064903 (2008).

8 De'bell, K. \& Lookman, T. Surface phase transitions in polymer systems. Rev. Mod. Phys. 65, 87 (1993).

9 Gong, Y. C. \& Wang, Y. M. Partitioning of polymers into pores near the critical adsorption point. Macromolecules 35, 7492 (2002)

10 Decase, R., Sommer, J.- U. \& Blumen, A. Static and dynamic properties of tethered chains at adsorbing surfaces: a Monte Carlo study. J. Chem. Phys. 120, 8831 (2004).

11 Janse van Rensburg, E. J. \& Rechnitzer, A. R. Multiple Markov chain Monte Carlo study of adsorbing self-avoiding walks in two and in three dimensions. J. Phys. A: Math. Gen. 37,6875 (2004).

12 Luo, M. B. The critical adsorption point of self-avoiding walks: a finite-size scaling approach. J. Chem. Phys. 128, 044912 (2008).

$13 \mathrm{Ma}$, L., Middlemiss, K. M., Torrie, G. M. \& Whittington, S. G. Location of the adsorption transition for polymers with excluded volume. Monte Carlo and enumeration results. J. Chem. Soc. Faraday Trans. // 74, 721 (1978).

14 Hammersly, J. M., Torrie, G. M. \& Whittington, S. G. Self-avoiding walks interacting with a surface. J. Phys. A: Math. Gen. 15, 539 (1982).

15 Eisenriegler, E., Kremer, K. \& Binder, K. Adsorption of polymer chains at surfaces: scaling and Monte Carlo analyses. J. Chem. Phys. 77, 6296 (1982).

16 Meirovitch, H. \& Livne, S. Computer simulation of long polymers adsorbed on a surface. II. Critical behavior of a single self-avoiding walk. J. Chem. Phys. 88, 4507 (1988).

17 Livne, S. \& Meirovitch, H. Computer simulation of long polymers adsorbed on a surface. I. Corrections to scaling in an ideal chain. J. Chem. Phys. 88, 4498 (1988).

18 Ishinabe, T. Critical exponents for surface interacting self-avoiding lattice walks. I. Three-dimensional lattices. J. Chem. Phys. 76, 5589 (1982).

19 Ziebarth, J. D., Wang, Y. M., Polotsky, A. \& Luo, M. B. Dependence of the critical adsorption point on surface and sequence disorders for self-avoiding walks interacting with a planar surface. Macromolecules 40, 3498 (2007).

20 Rosenbluth, M. N. \& Rosenbluth, A. W. Monte Carlo calculation of the average extension of molecular chains. J. Chem. Phys. 23, 356 (1955).

21 Gurler, M. T., Crabb, C. C., Dahlin, D. M. \& Kovac, J. Effect of bead movement rules on the relaxation of cubic lattice models of polymer chains. Macromolecules 16, 398 (1983).

22 Chen, Y. C. \& Luo, M. B. Monte Carlo study on the entropy of tail-like polymer chain with one end attached to flat surface. Int. J. Mod. Phys. B 21, 1787 (2007).

23 Mizutani, H. Determination of the end-to-end distance of high polymer molecule in solution. J. Phys. Soc. Jpn. 11, 245 (1956).

24 Yethiraj, A. Computer simulation study of two-dimensional polymer solutions. Macromolecules 36, 5854 (2003). 